\title{
Utilisation of Prophylactic Mastectomy in 10 European Centres
}

D.G.R. Evans ${ }^{1}$, E. Anderson ${ }^{2}$, F. Lalloo ${ }^{1}$, H. Vasen ${ }^{3}$, M. Beckmann ${ }^{4}$, D. Eccles ${ }^{5}$, S. Hodgson ${ }^{6}$, P. Møller ${ }^{7}$, J. Chang-Claude ${ }^{4}$, P. Morrison ${ }^{8}$, D. Stoppa-Lyonnet ${ }^{9}$, M. Steel ${ }^{2}$ and N. Haites ${ }^{10}$

${ }^{1}$ Department of Medical Genetics, St Mary's Hospital Manchester, UK

${ }^{3}$ Foundation for the Detection of Hereditary

Tumours, Leiden, Netherlands

${ }^{4}$ FrauenKlinik der Heinrich-Heine-

Universitat, Dusseldorf, Germany

${ }^{5}$ Wessex Regional Genetics Service,

Southampton, UK

${ }^{6}$ Division of Medical and Molecular

Genetics, Guy's Hospital, London, UK

${ }^{7}$ Unit of Medical Genetics, Norwegian

Radium Hospital, Oslo, Norway

${ }^{8}$ Department of Medical Genetics, Belfast

City Hospital, Belfast, UK

${ }^{9}$ Unité de Génétique Oncologique, Institut

Curie, Paris, France

${ }^{10}$ Department of Medical Genetics,

University of Aberdeen, Foresterhill,

Aberdeen, UK

\begin{abstract}
Increasingly women at high risk of breast cancer are opting for prophylactic surgery to reduce their risks. Data from 10 European centres that offer a risk counselling and screening service to women at risk show different approaches to the option of preventive surgery, although most centres adhere to a protocol including at least two risk counselling sessions and a psychological assessment. Thus far the combined centres have data on 174 women who have undergone prophylactic mastectomy with in excess of
\end{abstract}

400 women years of follow up. Operations were carried out on women with lifetime risks of $25-80 \%$, with an average annual expected incidence rate of $1 \%$ per women. No breast cancers have occurred in this cohort. Long term follow up on an extended group of women will be necessary to truly address the risk of subsequent breast cancer and the psychological sequelae.

\section{INTRODUCTION}

Management options available to women at high lifetime risk of breast cancer due to their family history, or to those women known to be carrying a mutation in BRCA1/2 (which confer a lifetime risk of breast cancer of $85 \%$ [1,2]) are limited. Screening with mammography is one option, as is prophylactic mastectomy. The efficacy of surgical procedures to reduce the risk of breast cancer is controversial [3,4], although it would appear that the residual risk of breast cancer is dependent upon the amount of breast tissue remaining following the surgical procedure. Recent work suggests that more women are considering prophylactic mastectomy $[5,6]$ and that protocols should be in place to deal with these requests. It has been suggested that surgery will increase life expectancy in BRCA1 or BRCA2 mutation carriers [7]. A recent study by Hartmann et al. [8] has demonstrated that women with a high risk of breast cancer can significantly reduce the incidence of the disease with prophylactic surgery. However, the level of reduction in those at highest risk (BRCA1/ BRCA2 carriers) is still unclear.

In view of this, our European collaboration has sought to develop protocols for women wishing 
to consider surgery and to assess the long-term outcomes in terms of cancer prevention and psychological sequelae.

\section{Genetic counselling and the family history clinic}

Family history clinics have been established at the ten participating centres (Table 1) between 1987 and 1992. They are administered by consultants in medical oncology, clinical genetics and breast surgery often with a multidisciplinary approach, with close involvement of radiologists, and a psychiatrist/psychologist.

At the initial appointment, a risk assessment is made from the family history using the Claus data [9]. Women at a lifetime risk of breast cancer of 1 in 6 or greater are offered mammography and clinical examination on a 1218 monthly basis between the ages of 30-35 and 50 years. If appropriate, genetic testing is discussed with women at this initial visit. Three of the centres (Manchester, Edinburgh, Heidelberg/Dusseldorf) routinely mention the possibility of prophylactic mastectomy to those women with a lifetime risk of 1 in 4 or greater. This is often done with a single sentence or a statement of the availability of the procedure as an option for prevention of breast cancer. This then allows women to extend the discussion if they wish to do so, or to state that they are not interested in surgery.

\section{THE PROPHYLACTIC MASTECTOMY PROTOCOL}

If women wish to discuss the procedure in greater detail, most centres offer a further appointment at least one month later. This gives women time to consider the procedure more fully and to discuss it with appropriate members of the family. Involvement of the partners in the decision making process is usually encouraged and they are, therefore, invited to attend each appointment. At the second appointment, with the geneticist or oncologist, a basic description of the surgery is given including the potential residual risk of various procedures. It is emphasised that the residual risk and complication rate may be higher if the surgery preserves the nipple/areolar complex. It is also usually made clear that these procedures are largely unproven $[3,4]$ as to their efficacy in reducing the risk of breast cancer. The patient is also challenged to consider the complications which may result in a potentially poor cosmetic result, as well as the possible impact upon her personal life and family dynamics.

The possibility of genetic testing is also discussed in terms of the availability of a living affected member of the family and the basic underlying structure of the family [10]. If possible, a time scale for genetic testing is discussed, and the woman is asked to consider the potential impact of having proceeded with

Table 1

Number and type of prophylactic mastectomies carried out by centre

\begin{tabular}{lrclrrrrr}
\hline Centre & No & Ages (years) & Mean & SM & MM & Bilat & Contralat & BRCA1/2 \\
\hline Manchester & 74 & $21-58$ & 40 & 18 & 56 & 69 & 5 & 11 \\
Edinburgh & 34 & $32-60$ & 43 & 0 & 34 & 15 & 19 & 2 \\
Aberdeen & 15 & $34-58$ & 43.5 & 5 & 10 & 13 & 2 & 3 \\
Leiden & 15 & $20-37$ & 36.5 & 8 & 7 & 15 & 0 & $?$ \\
Dusseldorf & 8 & $29-46$ & 38 & 3 & 5 & 8 & 0 & 1 \\
Oslo & 12 & $27-51$ & 39.5 & 6 & 6 & 11 & 1 & 0 \\
Southampton & 6 & $32-57$ & 43.5 & 4 & 2 & 3 & 3 & 0 \\
Belfast & 5 & $32-57$ & 40 & 5 & 0 & 2 & 3 & 1 \\
Guys & 5 & $28-37$ & 34 & 2 & 3 & 4 & 1 & 1 \\
Paris & 4 & $28-51$ & 40.5 & 2 & 2 & 1 & 3 & 0 \\
\hline
\end{tabular}

No - number; Bilat - bilateral; contralat - contralateral; SM - simple mastectomy; MM - modified mastectomy (includes subcutaneous). 
surgery if, later, she undergoes genetic testing which shows that she does not carry the causative mutation. Centres also emphasise that the genetic risk of breast cancer decreases with age and that the remaining risk of breast cancer if the women is older (over 40), is lower than the lifetime risk [9]. If a woman wishes to proceed, a psychological assessment is arranged. At this stage, confirmation of the breast cancers in the family are proactively sought by most centres if it has not already been completed. This ensures that the risk assessment is as accurate as possible. In one of our centres (Manchester) we have reported the presence of factitious histories within some families [11] and other centres, in confidence, confirm this experience.

After a psychological assessment, a more detailed surgical consultation is arranged to discuss the type of procedure best suited to the woman and whether a one or two stage procedure is preferable. This usually involves discussion of 3 basic options. The first; total bilateral mastectomy, the second; bilateral mastectomy with reconstruction, either with implants or tissue flaps, and the third; bilateral subcutaneous mastectomy which retains the overlying skin and nipple/areolar complex. The potential complications are also discussed along with the expected cosmetic result. Surgeons will usually have photographs available for women to view if they wish and may also arrange personal meetings with one or more patients who have already undergone bilateral mastectomy with reconstruction.

The whole process of consultations through to surgical procedure usually takes 6-12 months. This time delay is deliberate, in most centres, with the greatest delay at the beginning of the protocol in order to allow women time for the decision making process. If the protocol is run concurrently with a decision for predictive genetic testing, then the wait will generally be shorter. The number of women who are known to have undergone prophylactic surgery in each centre is shown in Table 1. The full protocol of two sessions at the family history clinic, a session with a psychiatrist and sessions with the surgeons was established in 1993 in Manchester. While only 2 other centres have a similar written protocol the remaining clinics generally adhere to the basic principles. The major difference is that several centres are mainly reactive, meaning that prophylactic surgery will usually be formally discussed only with women proven to be BRCA1/2 mutation carriers. No centre actively recommends the procedure even in the latter category.

There is no clear pattern in terms of the surgical procedure recommended in women who have decided on surgery. While some units are cautious about offering skin/nipple-preserving mastectomies, these options are generally available in every case.

\section{FOLLOW-UP}

Follow up of women who have undergone surgery is considered an important part of the protocol in each centre. In Manchester, women who have undergone prophylactic mastectomy are followed up annually at a "multidisciplinary day". As well as discussion of problems/issues with all the relevant clinicians (Geneticist/ oncologist/psychiatrist/surgeons), each patient is examined. Clinical examination by palpation of the breasts is felt to be adequate as remaining breast tissue is very superficial in all types of surgical procedure. Thus far, no cancers have occurred prospectively in the 174 women who have undergone surgery. Using data from Claus [9] and the International Linkage Consortium [1] it is possible to calculate annual age dependant expected incidence rates for breast cancer in women who have not undergone surgery. The mean expected rate for our cohort of women is $1 \%$ annually, reflecting a lifetime risk that ranged from $25-80 \%$. Even though this cohort already has a follow up in excess of 400 woman years, only 4 cancers would have been expected. Follow up of an extended cohort for more than 5 years will be necessary to address the issue of risk reduction. If this is to be analysed by type of surgery or by confining to known BRCA1/2 mutation carriers, (currently only 17/174) even longer follow up will be necessary. 


\section{References}

[1] Ford, D., Easton, D.F., Bishop, D.T., Narod, S.A. and Goldgar, D.E. Risks of cancer in BRCA1mutation carriers. Lancet 343, (1994) 692-695.

[2] Wooster, R., Neuhausen, S.L., Mangion, J. et al. Localization of a breast cancer susceptibility gene, BRCA2, to chromosome 13q12-13. Science 265, (1994) 2088-2090.

[3] Goodnight, J.E., Quagliana, J.M. and Morton, D.L. Failure of subcutaneous mastectomy to prevent the development of breast cancer. $J$. Surg. Oncol. 26, (1984) 198-201.

[4] Zeigler, L.D. and Kroll, S.S. Primary breast cancer after prophylactic mastectomy. Am. J. Clin. Oncol. 14, (1991) 451-454.

[5] Lynch, H.T., Lemon, S.J., Durham, C. et al. A descriptive study of BRCA1 testing and reactions to disclosure of test results. Cancer 79, (1997) 2219-2228.

[6] Eeles, R., Cole, T., Taylor, R., Lunt, P. and Baum, M. Prophylactic mastectomy for genetic predisposistion to breast cancer: the proband's story. Clin. Oncol. 8, (1996) 222-225.

[7] Schrag, D., Kuntz, K.M., Garbor, J.E. and Weeks, J.C. Decision analysis - effects of prophylactic mastectomy and oophorectomy on life expectancy among women with BRCA1 or BRCA2 mutations. N. Engl. J. Med. 336, (1997) 1465-1471.

[8] Hartmann, L.C., Schiad, D.J., Woods, J.E. et al. Efficacy of bilateral prophylactic mastectomy in women with a family history of breast cancer. $N$. Engl. J. Med. 340, (1999) 77-84.

[9] Claus, E.B., Risch, N. and Thompson, W.D. Autosomal dominant inheritance of early-onset breast cancer; implications for risk prediction. Cancer 73, (1994) 643-651.

[10] Eeles, R. Testing for the breast cancer predisposition gene, BRCA1. BMJ 313, (1996) 572-573.

[11] Evans, D.G.R., Kerr, B., Cade, D., Hoare, E. and Hopwood, P. Fictitious breast cancer family history. Lancet 348, (1996) 1034. 


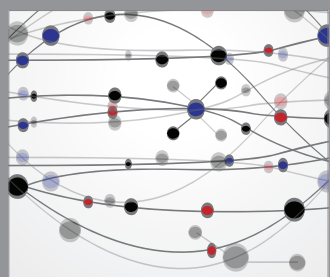

The Scientific World Journal
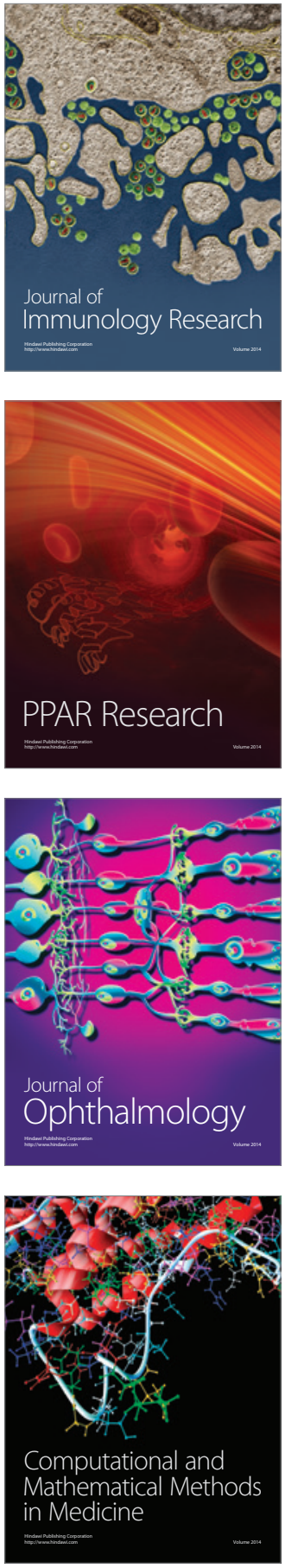

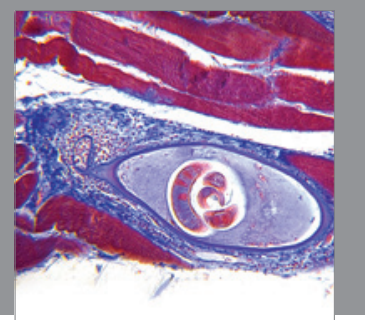

Gastroenterology

Research and Practice
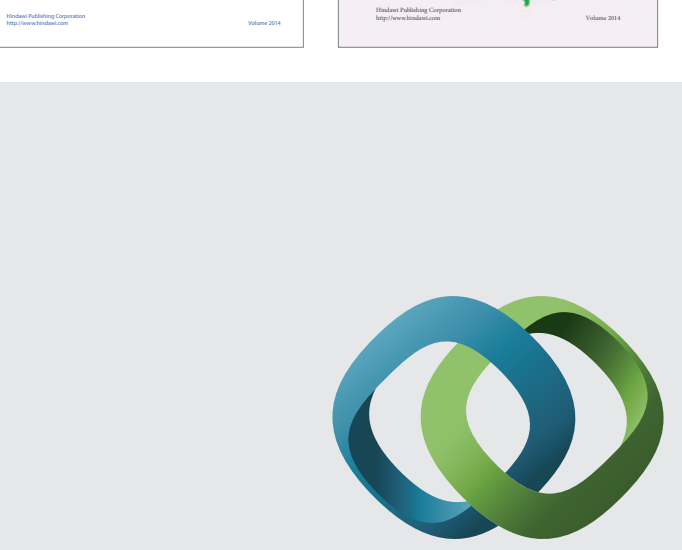

\section{Hindawi}

Submit your manuscripts at

http://www.hindawi.com
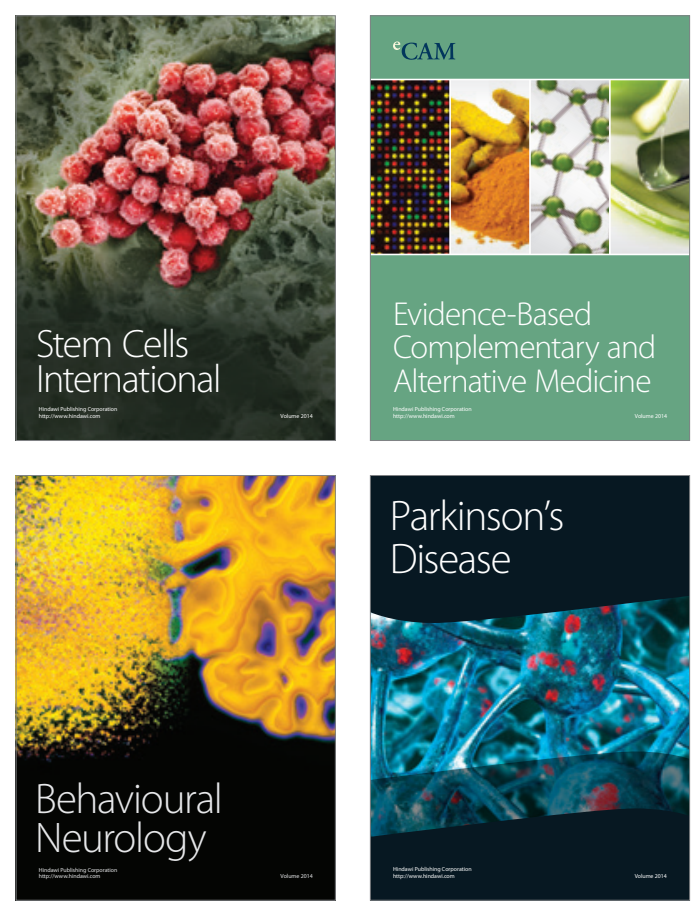

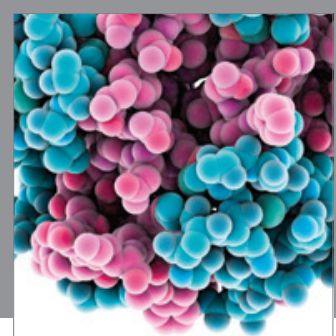

Journal of
Diabetes Research

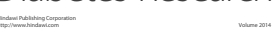

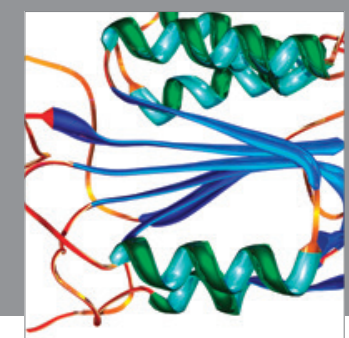

Disease Markers
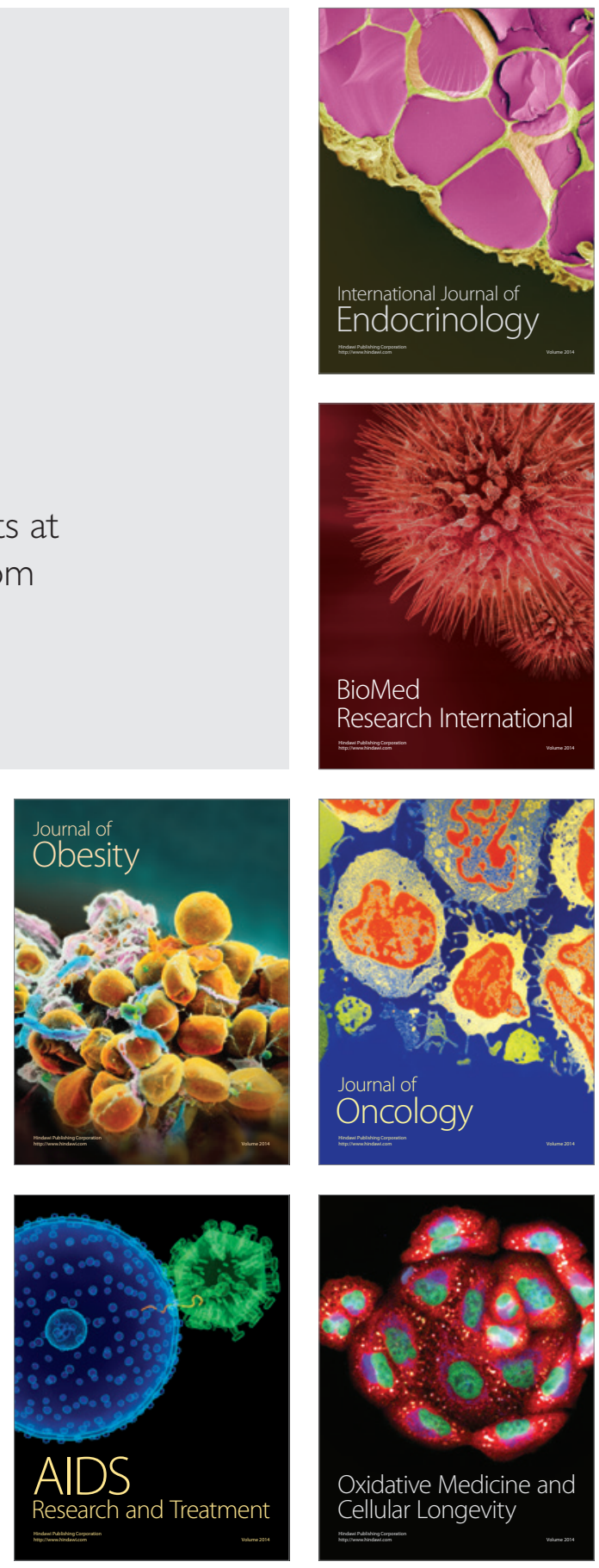